\title{
Medium-Term Earthquake Forecast Using Gravity Monitoring Data: Evidence from the Yutian and Wenchuan Earthquakes in China
}

\author{
Yiqing Zhu' ${ }^{1,2}$ and F. Benjamin Zhan ${ }^{3,4}$ \\ ${ }^{1}$ Institute of Geodesy and Geophysics, Chinese Academy of Sciences, Wuhan 430077, China \\ ${ }^{2}$ Second Crust Monitoring and Application Center, China Earthquake Administration, Xi'an 710054, China \\ ${ }^{3}$ School of Resource and Environmental Science, Wuhan University, Wuhan 430079, China \\ ${ }^{4}$ Texas Center for Geographic Information Science, Department of Geography, Texas State University, San Marcos, TX 78666, USA
}

Correspondence should be addressed to F. Benjamin Zhan, zhan@txstate.edu

Received 15 July 2011; Accepted 28 December 2011

Academic Editor: Jiancang Zhuang

Copyright ( $) 2012$ Y. Zhu and F. B. Zhan. This is an open access article distributed under the Creative Commons Attribution License, which permits unrestricted use, distribution, and reproduction in any medium, provided the original work is properly cited.

Gravity changes derived from regional gravity monitoring data in China from 1998 to 2005 exhibited noticeable variations before the occurrence of two large earthquakes in 2008 in China-the 2008 Yutian (Xinjiang) $M_{s}=7.3$ earthquake and the 2008 Wenchuan (Sichuan) $M_{s}=8.0$ earthquake. Based on these gravity variations, a group of researchers at the Second Crust Monitoring and Application Center of China Earthquake Administration made a suggestion in December of 2006 that the possibility for the Yutian (Xinjiang) and Wenchuan (Sichuan) areas to experience a large earthquake in either 2007 or 2008 was high. We review the gravity monitoring data and methods upon which the researchers reached these medium-term earthquake forecasts. Experience related to the medium-term forecasts of the Yutian and Wenchuan earthquakes suggests that gravity changes derived from regional gravity monitoring data could potentially be a useful medium-term precursor of large earthquakes, but significant additional research is needed to validate and evaluate this hypothesis.

\section{Introduction}

In December 2006, a group of researchers at the Second Crust Monitoring and Application Center of China Earthquake Administration suggested that the possibility for the areas surrounding Yutian (Xinjiang) and Wenchuan (Sichian) to experience a large earthquake in either 2007 or 2008 was high [1]. The forecasted location, magnitude, and timeframe of the two earthquakes are summarized in Table 1. These researchers used gravity changes derived from regional gravity monitoring data in China from 1998 to 2005 as the primary precursory information to make these suggestions. In this paper, we first review ground gravity surveys conducted in China in 1998, 2000, 2002, and 2005 and then report how the researchers used gravity monitoring data to make medium-term (less than three years) forecasts of the Yutian and Wenchuan earthquakes. In addition, we make a few observations about possible connections between gravity changes and the occurrence of a large earthquake and provide some discussions about future research directions in earthquake research using gravity monitoring data.

\section{Nationwide Gravity Survey Campaigns in China}

China began establishing an ambitious countrywide crustal movement observation network in 1998-Crustal Movement Observation Network of China (CMONOC) (Figure 1). Among other types of observation stations, the network consisted of 25 absolute gravity observation stations and 360 relative gravity observation stations at the end of 2005. The absolute gravity observation stations serve as a control network through which a stable and uniform gravity field throughout the country can be computed. Gravity field dynamics throughout the country then can be determined 


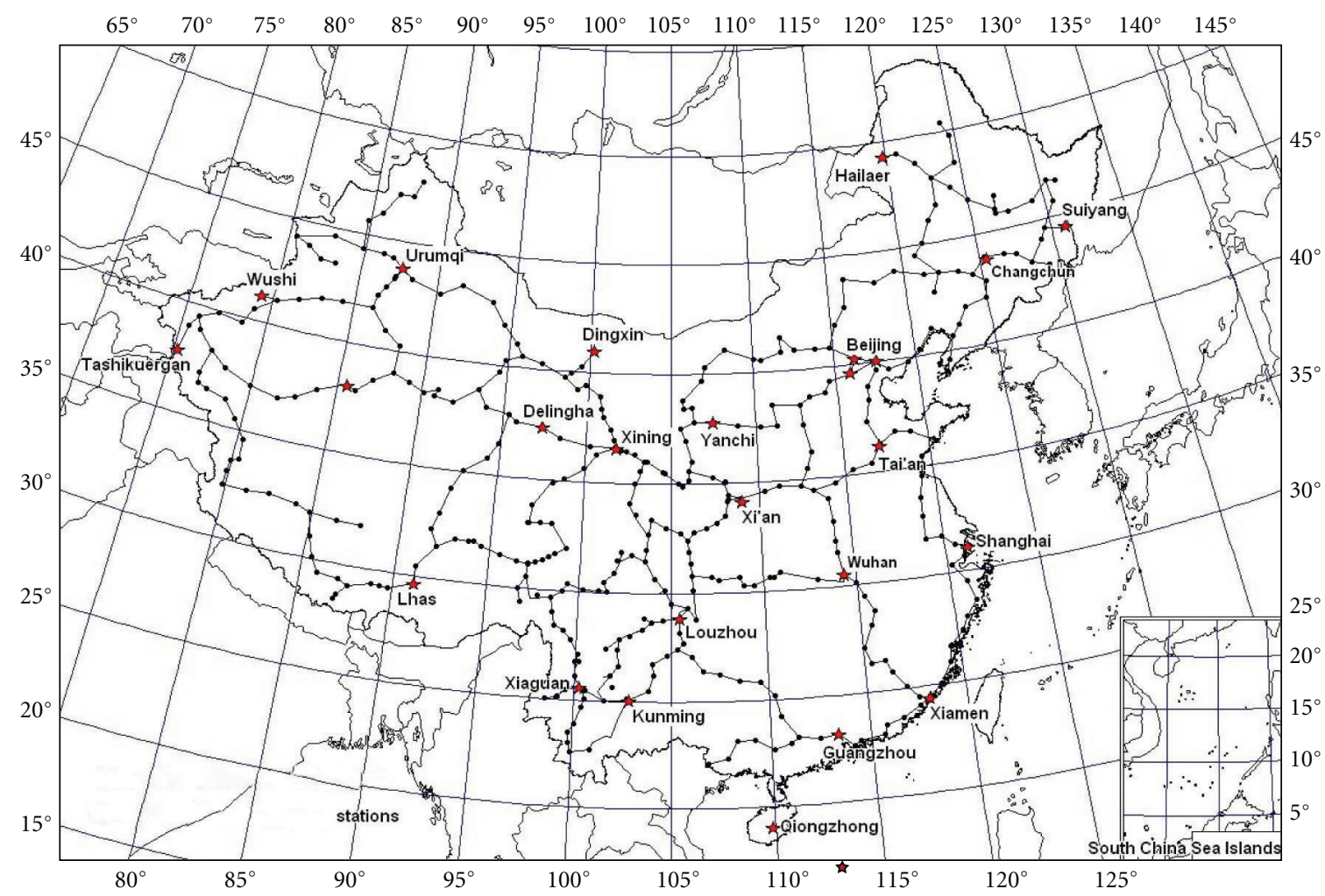

Absolute gravity measurement stations

- Relative gravity measurement stations

Figure 1: Crustal Movement Observation Network of China (CMONOC).

TABLE 1: Summary of the three elements of the two forecasted and actual earthquakes.

\begin{tabular}{lcc}
\hline Earthquake parameters & Forecasted & Actual \\
\hline \multicolumn{3}{c}{ The Yutian earthquake $[1,4]$} \\
\hline Epicenter & $36.0^{\circ} \mathrm{N}, 80.0^{\circ} \mathrm{E}$ & $35.6^{\circ} \mathrm{N}, 81.6^{\circ} \mathrm{E}$ \\
Magnitude & $\mathrm{Ms} 6.0-\mathrm{Ms} 7.0$ & $\mathrm{Ms7.3}$ \\
Timeframe & $2007-2008$ & March 21,2008 \\
\hline \multicolumn{3}{c}{ The Wenchuan earthquake $[1,5]$} \\
\hline Epicenter & $31.6^{\circ} \mathrm{N}, 103.7^{\circ} \mathrm{E}$ & $31.0^{\circ} \mathrm{N}, 103.4^{\circ} \mathrm{E}$ \\
Magnitude & $\mathrm{Ms} 6.0-\mathrm{Ms} 7.0$ & $\mathrm{Ms} 8.0$ \\
Timeframe & $2007-2008$ & May 12,2008 \\
\hline
\end{tabular}

through repeated mobile gravity surveys at the 360 relative gravity stations. China Earthquake Administration, the Chinese Academy of Sciences, and China State Bureau of Surveying and Mapping coordinated four rounds of nationwide ground gravity surveys in 1998, 2000, 2002, and 2005 . Because details of the gravity surveys and data processing are provided in two recent papers $[2,3]$, we only briefly describe the surveys as well as procedures for data processing in the rest of this section and in the next section.

Surveyors with significant field observation experience from three different organizations - the Institute of Geodesy and Geophysics of the Chinese Academy of Sciences, the Institute of Seismology of China Earthquake Administration, and the State Bureau of Surveying and Mapping in Chinaconducted the absolute gravity surveys using FG-5 gravimeters. The accuracy of the observed absolute gravity survey at each station was higher than $5 \times 10^{-8} \mathrm{~m} / \mathrm{s}^{2}[2,6,7]$.

After field survey, absolute gravity data at the absolute gravity observation stations were adjusted for earth-tide, speed of light, local air pressure, polar motion, and vertical gradient. The adjusted data then were used for subsequent analysis and integration with the relative gravity survey data. Both the absolute and relative gravity surveys were all conducted during the months from July through November in 1998, 2000, 2002, and 2005. This arrangement of conducting the gravity surveys in the same months of different years was designed to reduce possible seasonal hydrological effects on gravity observed at the same location in different years.

The mobile relative gravity surveys were completed through joint efforts by two organizations of China Earthquake Administration - the Institute of Seismology and the Second Crust Monitoring and Application Center. Surveyors used the LaCoste and Romberg gravimeters (model G (LCR$\mathrm{G})$ ) in the relative gravity surveys. The accuracy of LCR-G gravimeters was higher than $10 \times 10^{-8} \mathrm{~m} / \mathrm{s}^{2}$, and the drifts of null reading values of the LCR-G gravimeters were within $\pm 5 \times 10^{-8} \mathrm{~ms}^{-2}$ /hour. Field surveyors followed the Chinese national field work procedures and guidelines when conducting the mobile relative gravity surveys to ensure that the relative gravity survey data were in the best possible quality $[2,6,7]$. 


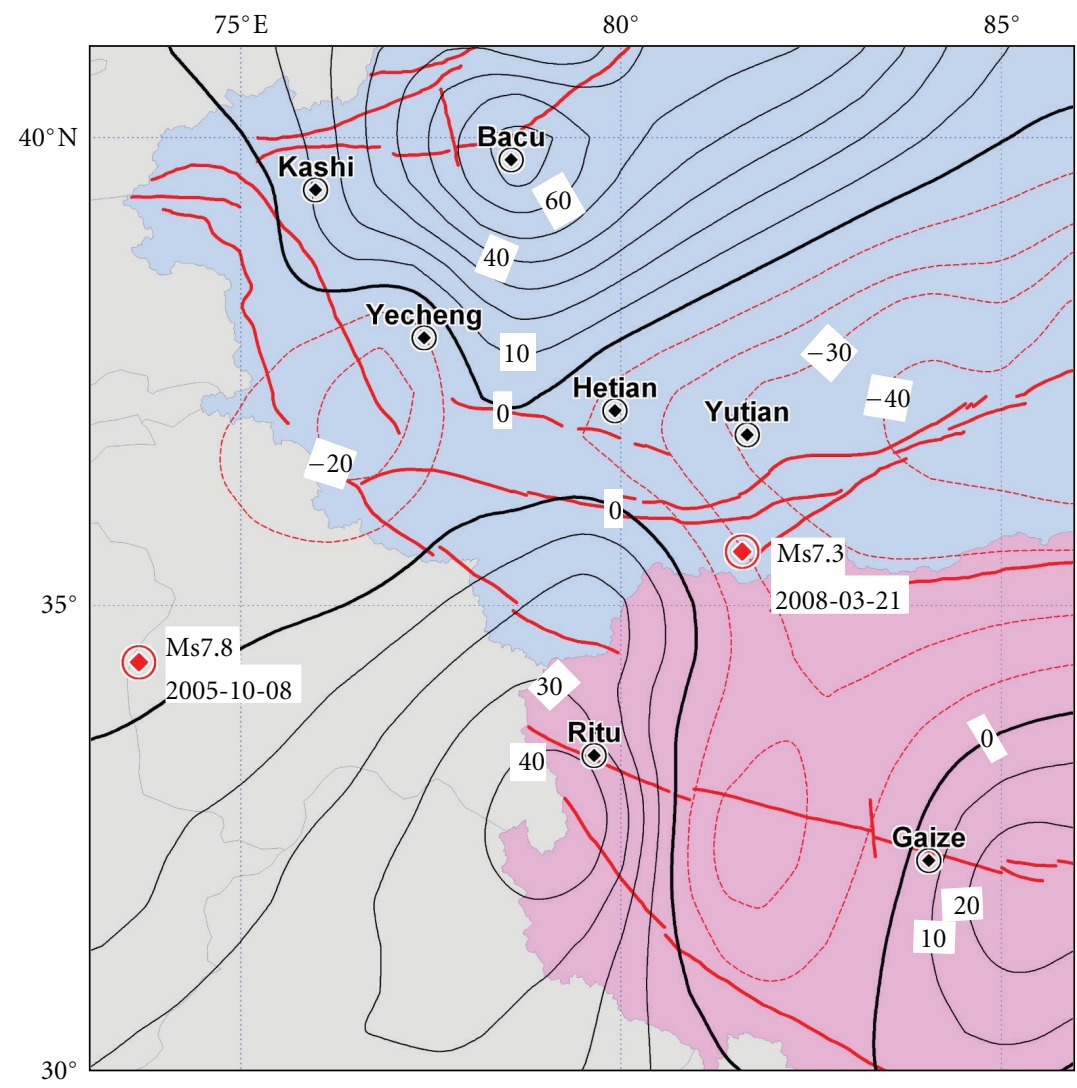

FIGURE 2: A contour map showing gravity changes (in $10^{-8} \mathrm{~m} / \mathrm{s}^{2}$ ) from 1998 to 2005 before the Yutian Earthquake. The contours on the map were produced using a resolution of 0.25 geographic degrees.

\section{Processing of Field Gravity Survey Data}

The key in processing the field gravity data described previously is to integrate the high precision absolute gravity survey data with the mobile relative gravity survey data and then compute the absolute gravity at each of the relative gravity observation stations. A 3-step procedure was used to determine the final gravity data at each relative gravity observation station. First, relative gravity survey data were adjusted for solid earth-tide, air pressure, first-order item, and height of gravimeters. Second, a preliminary analysis of the gravity survey data across all four rounds of surveys was conducted to eliminate data with possible gross errors. Third, the Adjustment Program for Mobile Gravimetric Data Measured by LaCoste and Romberg Gravimeters (LGADJ) software package recommended by China Earthquake Administration was used to obtain the (absolute) gravity data at the location of each observation station for each of the four time periods. The LGADJ software package is a standard computational tool that can be used to integrate absolute and relative gravity survey data to obtain the final absolute gravity data at each of the relative gravity observation stations $[8,9]$. The average accuracy of the final adjusted gravity data at each relative observation station was better than $15 \times 10^{-8} \mathrm{~m} / \mathrm{s}^{2}$ across all four years.

Least-squares collocation was used to calculate gravity data at the intersections of a grid with a resolution of 0.25 geographic degrees in the region in question based on the computed gravity data at the observation stations mentioned above. The least-squares collocation method treats observed gravity data at the observation stations and gravity data to be estimated at other locations on the grid as random variables. The method provides the best possible estimation of ground gravity data at locations where observation data did not exist. After obtaining the gravity data on the grid, the MapGIS software was used to produce the contour maps of gravity changes between different time periods.

\section{Gravity Changes before the Yutian Earthquake}

As can be seen from Figure 2, there were significant gravity changes in the region shown in Figure 2 from 1998 to 2005. Based on empirical experience, when the difference between the largest positive gravity change and the highest negative gravity change in the region in question exceeds $80 \times$ $10^{-8} \mathrm{~m} / \mathrm{s}^{2}$, the gravity changes in the region are considered significant. The gravity change gradient extended from the southwest part of the region shown in Figure 2 to the northeast part of the region. Positive gravity changes occurred in the southwest part of the region, and negative gravity changes took place in the northeast part of the region. The difference between the positive and negative gravity changes was as great as $80 \times 10^{-8} \mathrm{~m} / \mathrm{s}^{2}$. Based on the contour map shown in Figure 2, a belt with a steep gravity change gradient can be identified. This belt extended from Yutian to Hetian. 


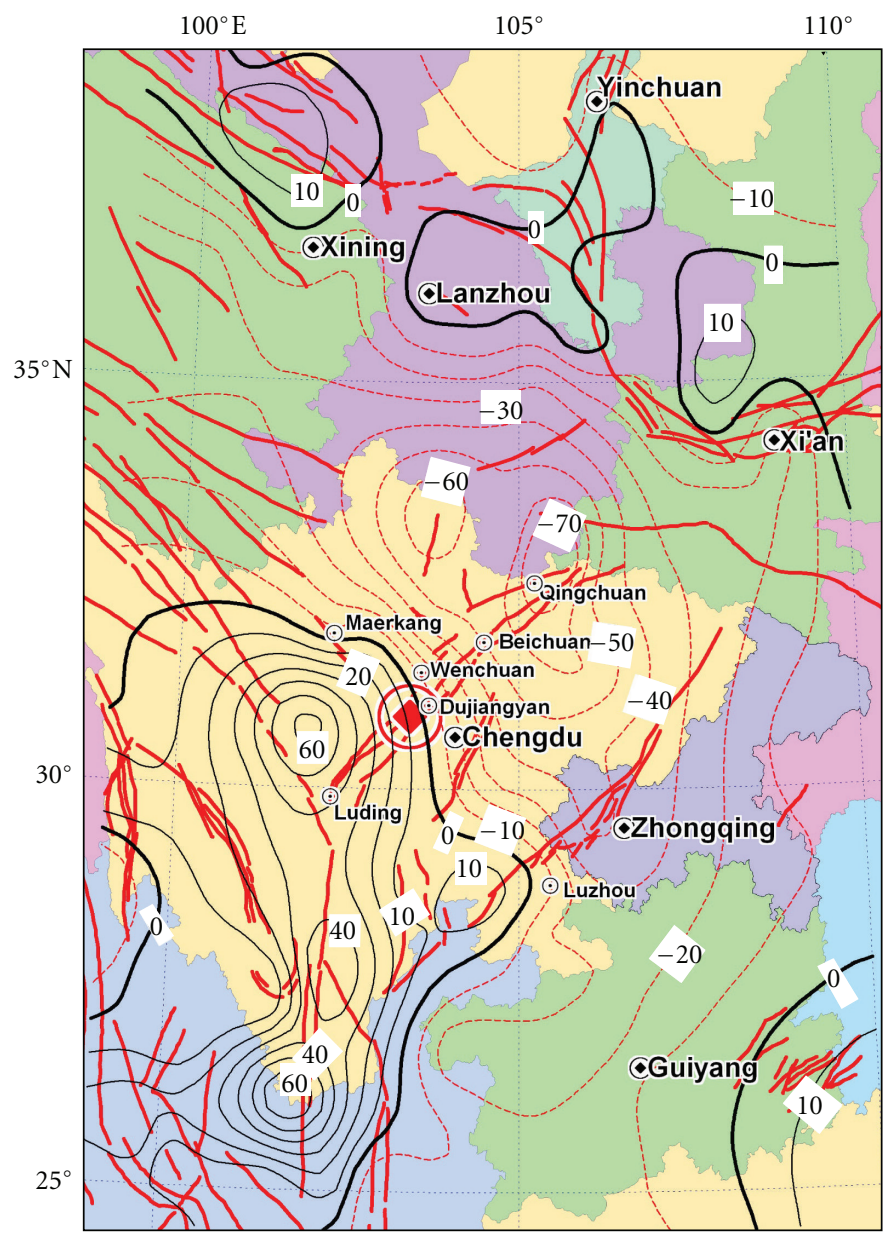

Figure 3: A contour map illustrating gravity changes from 1998 to 2005 before the Wenchuan earthquake. The contours on the map were produced using a resolution of 0.25 geographic degrees.

\section{Gravity Changes before the Wenchuan Earthquake}

Characteristics of gravity changes from 1998 to 2005 before the Wenchuan earthquake are shown in Figure 3. Within the region, the rhombus-shaped Chuan-Dian (Sichuan-Yunnan) Plate showed positive gravity changes from 1998 to 2005 as great as $60 \times 10^{-8} \mathrm{~m} / \mathrm{s}^{2}$, whereas areas surrounding the Chuan-Dian Plate exhibited negative gravity changes during the same period with a negative peak gravity change value as large as $60 \times 10^{-8} \mathrm{~m} / \mathrm{s}^{2}$. These positive and negative gravity changes and their differences were particularly evident in northern Sichuan where the difference of gravity changes was as great as $60 \times 10^{-8} \mathrm{~m} / \mathrm{s}^{2}$. This difference was particularly evident in a belt with a steep gravity change gradient. This belt extended from Luzhou, through Wenchuan, to Maerkang (Figure 3).

\section{Medium-Term Forecasts of the Yutian and Wenchuan Earthquakes}

As stated at the beginning of this paper, the forecast location, magnitude, and timeframe of the Yutian and Wenchuan earthquakes are summarized in Table 1. For the Yutian earthquake [1], the location of the earthquake was determined based on analysis results of past earthquakes and an observation that an earthquake typically occurs at the intersection of the zero gravity change contour line in the area with high gravity change gradients and the fault that is mostly likely to accumulate sufficient energy for a major earthquake, the Kangxiwar-Altyn Tagh Fault in this case. The magnitude of the Yutian earthquake was estimated to be between $M_{s}=6.0$ and $M_{s}=7.0$ based on the highest value $\left(60 \times 10^{-8} \mathrm{~m} / \mathrm{s}^{2}\right)$ of the differences of gravity changes in the region from 1998 to 2005 (Figure 2). Again, this estimation was based on empirical data about the relationships between the magnitudes of earthquakes occurred before the Yutian earthquake and values of the differences of gravity changes before these past earthquakes. The determination of the timeframe of the forecasted Yutian earthquake was also based on past experience and empirical data. Earthquakes typically occur within one to two years after a period of significant gravity changes in the region in question. Because the region experienced significant gravity changes from 1998 to 2005, it was forecasted that the earthquake would occur sometime in 2007 or 2008 . 
TABLE 2: Nine large $\left(M_{s} \geq 6.8\right)$ earthquakes occurred within or near China from 2001 to 2008 and gravity changes before these nine earthquakes as detected by the Crustal Monitoring Network of China (CMONOC) [3].

\begin{tabular}{|c|c|c|c|c|c|}
\hline ID & $\begin{array}{l}\text { Earthquake } \\
\text { (province or equivalent) }\end{array}$ & Magnitude $\left(M_{s}\right)$ & $\begin{array}{c}\text { Location of } \\
\text { actual epicenter }\end{array}$ & $\begin{array}{c}\text { Date of } \\
\text { earthquake }\end{array}$ & $\begin{array}{l}\text { Observed peak-to-valley difference of gravity changes } \\
\left.\text { (in } 10^{-8} \mathrm{~m} / \mathrm{s}^{2}\right) \text { (time period) and region of change }\end{array}$ \\
\hline 1 & Kunlun (Xinjiang) & 8.1 & $36.2^{\circ} \mathrm{N}, 90.9^{\circ} \mathrm{E}$ & 14-Nov-2001 & $\begin{array}{l}130 \text { (1998-2000); bordering areas between Qinghai and } \\
\text { Xinjiang }\end{array}$ \\
\hline 2 & Offshore east of Taiwan & 7.5 & $24.4^{\circ} \mathrm{N}, 122.1^{\circ} \mathrm{E}$ & 31-Mar-2002 & 80 (1998-2000); coastal area in Fujian facing Taiwan \\
\hline 3 & Wangqing (Jilin) & 7.2 & $43.5^{\circ} \mathrm{N}, 103.6^{\circ} \mathrm{E}$ & 29-Jun-2002 & $\begin{array}{l}60 \text { (1998-2000); the Wangqing-Changchun-Suiyang } \\
\text { area in Jilin }\end{array}$ \\
\hline 4 & Jashi (Xinjiang) & 6.8 & $39.5^{\circ} \mathrm{N}, 77.2^{\circ} \mathrm{E}$ & 24-Feb-2003 & $\begin{array}{l}60 \text { (1998-2000); the Kashi-Wushi-Kuerle area in } \\
\text { southwest Xinjiang }\end{array}$ \\
\hline 5 & $\begin{array}{l}\text { The bordering areas } \\
\text { between China and } \\
\text { Russia near Northern } \\
\text { Xinjiang }\end{array}$ & 7.9 & $49.9^{\circ} \mathrm{N}, 87.9^{\circ} \mathrm{E}$ & 28-Sep-2003 & 60 (2000-2002); northern Xinjiang \\
\hline 6 & Gaizhe (Tibet) & 6.9 & $32.5^{\circ} \mathrm{N}, 85.2^{\circ} \mathrm{E}$ & 9-Jan-2008 & 80 (2002-2005); the Gaize and Nima area in Tibet \\
\hline 7 & Yutian (Xinjiang) & 7.3 & $35.6^{\circ} \mathrm{N}, 81.6^{\circ} \mathrm{E}$ & 21-Mar-2008 & 90 (2002-2005); the Yutian and Hetian area in Xinjiang \\
\hline 8 & Wenchuan (Sichuan) & 8.0 & $31.0^{\circ} \mathrm{N}, 103.4^{\circ} \mathrm{E}$ & 12-May-2008 & $\begin{array}{l}130 \text { (1998-2005); northern Sichuan along } \\
\text { Luzhou-Wenchuan-Maerkang }\end{array}$ \\
\hline 9 & Zhongba (Tibet) & 6.8 & $31.0^{\circ} \mathrm{N}, 83.6^{\circ} \mathrm{E}$ & 25-Aug-2008 & 90 (2002-2005); the Zhongba and Nima area in Tibet \\
\hline
\end{tabular}

A similar procedure was used to forecast the Wenchuan earthquake $[1,5]$. The forecasted location of the Wenchuan earthquake was determined to be at the intersection of the Longmenshan Fault and the zero gravity change line shown in Figure 3. The magnitude was forecasted to be between $M_{s}=6.0$ and $M_{s}=7.0$ based on the highest value of the differences of gravity changes in the region. The timeframe-sometime in 2007 or 2008 - was estimated based on the observation that the earthquake would occur in about one to two years from 2005 after a period of significant gravity changes from 1998 to 2005 . Again, this timeframe of the earthquake forecast was based on empirical data and experience of the researchers. Additional research is needed to remove the subjective nature in the determination of the timeframe of a forecasted earthquake.

\section{Conclusions and Discussions}

Despite many years of research, short- to medium-term earthquake forecast has remained a difficult task [10-12]. Although the potential of using gravity data for earthquake research was recognized nearly 50 years ago [13-20], there existed few cases demonstrating the usefulness of gravity monitoring data for useful earthquake forecast. Based on discussions presented above, we argue that information derived from large-scale regional gravity monitoring data has the potential to be used as a precursor for forecasting large earthquakes in a medium-term of no more than five years, particularly when that information is combined with expert knowledge about historic seismic activities, seismicity rate, as well as geological and tectonic conditions in the region in question.

The benefits of medium-term earthquake forecast are obvious. At a minimum, the forecast will help delineate high risk areas within a short timeframe of usually two to three years, enable decision makers to deploy limited resources to targeted areas, and hopefully help save lives and protect properties. An immediate need in advancing this line of research is to support and continue empirical research for medium-term earthquake forecasts using gravity monitoring data at a regional scale. We advocate that countries in highrisk earthquake zones establish regional scale gravity monitoring networks in areas covering these earthquake zones. A procedure similar to the one used in China can be employed to periodically collect ground gravity survey data [4-7]. These procedures can be modified, improved, and replicated to suite situations in other parts of the world.

Gravity data reported in this paper suffer a number of limitations. First, there are several uncertainties in the data that affect the usefulness and reliability of the gravity changes derived from the gravity monitoring data. These uncertainties include gravity variations due to hydrological effects and vertical crustal displacements, as well as inevitable errors in ground gravity surveys across a large region. Second, the density of the monitoring network as measured by the number of gravity observation stations was low. This low density undoubtedly would affect the results of the gravity monitoring data. Third, the time interval of the ground gravity survey was two or three years in the four rounds of gravity surveys discussed in this paper. Gravity monitoring data of the same nature with higher frequency would improve the usefulness of the data. Future efforts of data collection and data processing should strive to reduce the uncertainties and limitations mentioned above.

There are a number of significant research questions that warrant additional research. The first question is whether there were significant gravity changes before other large earthquakes in China and its neighboring regions during the same time period. A recent article by Zhan and his colleagues demonstrated that significant gravity changes were observed before all nine large earthquakes that ruptured within or near mainland China from 2001 to 2008 [3]. Table 2 provides 
a summary of the significant gravity changes before these nine earthquakes. However, it remains to be evaluated whether these gravity changes are indeed associated with the large earthquakes and if similar observations can be made in other parts of the world.

The second research question is how to remove the subjective nature in the determination of the magnitude, location, and timeframe of a forecasted large earthquake. The forecast examples presented in this paper heavily relied on subjective expert knowledge and empirical experience of the researchers. What is missing is a set of objective computational procedures that are generally applicable to other parts of the world for determining the magnitude, location, and timeframe of a pending large earthquake [21].

Third, once the method is fully developed, the statistical significance of gravity changes and their relationships with the occurrence of large earthquakes will have to be evaluated using historical data. In addition, a quest of enormous value is to investigate the physical processes that lead to gravity changes before large earthquakes and determine how those physical processes may be used in earthquake forecast. Progresses about research addressing the questions stated above will be reported in future publications.

\section{Acknowledgments}

Most of this paper was written in the summers of 2009 and 2010 while F. B. Zhan. was visiting Wuhan University in China. He appreciates the support from the Chang Jiang Scholars Award Program and Wuhan University. Y. Zhu's work was in part supported by a grant from the National Science Foundation of China (no. 40874035) and by a special earthquake research project grant (no. 200908029) from China Earthquake Administration.

\section{References}

[1] Y. Zhu, W. Liang, Y. Xu, and L. Liu, "Spatial-temporal characteristics of gravity field dynamics in mainland China and forecast of earthquake trends," in Annual Research Report about Earthquake Trends in China in 2007, pp. 111-119, Second Crust Monitoring and Application Center, China Earthquake Administration, Xi'An, China, 2010.

[2] Y. Zhu, F. B. Zhan, J. Zhou, W. Liang, and Y. Xu, "Gravity measurements and their variations before the 2008 Wenchuan earthquake," Bulletin of the Seismological Society of America, vol. 100, no. 5, pp. 2815-2824, 2010.

[3] F. B. Zhan, Y. Zhu, J. Ning, J. Zhou, W. Liang, and Y. Xu, "Gravity changes before large earthquakes in China: 1998-2005," Geo-Spatial Information Science, vol. 14, no. 1, pp. 1-9, 2011.

[4] Y. Zhu, Y. Xu, and W. Liang, "Medium-term prediction of Yutian, Xinjiang $M_{s}=7.3$ earthquake in 2008," Journal of Geodesy and Geodynamics, vol. 28, no. 5, pp. 13-15, 2008 (Chinese).

[5] Y. Zhu, W. Liang, and Y. Xu, "Medium-term prediction of $M_{s}=8.0$ earthquake in Wenchuan, Sichuan by mobile gravity," Recent Developments in World Seismology, vol. 7, pp. 36-39, 2008 (Chinese).

[6] H. Xu, "Function of gravimetry in CMONOC," Journal of Geodesy and Geodynamics, vol. 23, pp. 1-3, 2003 (Chinese).
[7] W. M. Zhang, Y. Wang, and X.-H. Zhou, "Expectation and application study of absolute gravity observation technology in China," Progress in Geophysics, vol. 23, no. 1, pp. 69-72, 2008 (Chinese).

[8] D. Liu, H. Li, and S. Liu, "A management and analysis system of gravity survey data-LGADJ," in Proceedings of the Research Symposium of Application of Seismological Prediction Methods, pp. 339-350, Seismological Press, Beijing, China, 1991.

[9] X. Wu, Y. Chen, H. Li et al., "National network of gravity measurement and assessment of its accuracy," Earthquake Research of China, vol. 11, no. 1, pp. 92-98, 1995 (Chinese).

[10] R. J. Geller, "Earthquake prediction: a critical review," Geophysical Journal International, vol. 131, no. 3, pp. 425-450, 1997.

[11] R. J. Geller, D. D. Jackson, Y. Y. Kagan, and F. Mulargia, "Geoscience-earthquakes cannot be predicted," Science, vol. 275, no. 5306, pp. 1616-1617, 1997.

[12] M. Wyss and D. C. Booth, "The IASPEI procedure for the evaluation of earthquake precursors," Geophysical Journal International, vol. 131, no. 3, pp. 423-424, 1997.

[13] H. W. Oliver, M. F. Kane, and L. C. Pakiser, "Gravity anomalies in Central Sierra Nevada, California," Journal of Geophysical Research, vol. 66, pp. 4265-4271, 1961.

[14] D. F. Barnes, "Gravity changes during Alaska earthquake," Journal of Geophysical Research, vol. 71, pp. 451-456, 1966.

[15] R. Page, "Aftershocks and microaftershocks of Great Alaska earthquake of 1964," Bulletin of the Seismological Society of America, vol. 58, pp. 1131-1168, 1968.

[16] Y.-T. Chen, H.-D. Gu, and Z.-X. Lu, "Variations of gravity before and after the Haicheng earthquake, 1975, and the Tangshan earthquake, 1976," Physics of the Earth and Planetary Interiors, vol. 18, no. 4, pp. 330-338, 1979.

[17] M. H. Wei, W. Zhao, and L. Ma, "Gravity Changes before and After the Tangshan earthquake of July 28, 1976, and Possible Interpretation," Journal of Geophysical Research-Solid Earth and Planets, vol. 90, pp. 5421-5428, 1985.

[18] G. Gu, J. T. Kuo, K. Liu, J. Zheng, H. Lu, and D. Liu, "Seismogenesis and occurrence of earthquakes as observed by temporally continuous gravity variations in China," Chinese Science Bulletin, vol. 43, no. 1, pp. 8-21, 1998.

[19] J. T. Kuo, J. H. Zheng, S. H. Song, and K. R. Liu, "Determination of earthquake epicentroids by inversion of gravity variation data in the BTTZ region, China," Tectonophysics, vol. 312, no. 2-4, pp. 267-281, 1999.

[20] K. R. Liu, J. X. Zheng, J. T. Kuo et al., "Mobile gravity survey and the combined dilatation model (CDM) in the BTTZ region," in Advances in Pure and Applied Geophysics, pp. 167178, Meteorological Publishing House, Beijing, China, 2002.

[21] F. B. Zhan, Y. Zhu, M. F. Goodchild, and J. Zhou, "An approach for identifying high risk areas of medium-term large earthquakes using gravity monitoring data," Tech. Rep., Department of Geography, Texas Center for Geographic Information Science, Texas State University-San Marcos, 2010. 

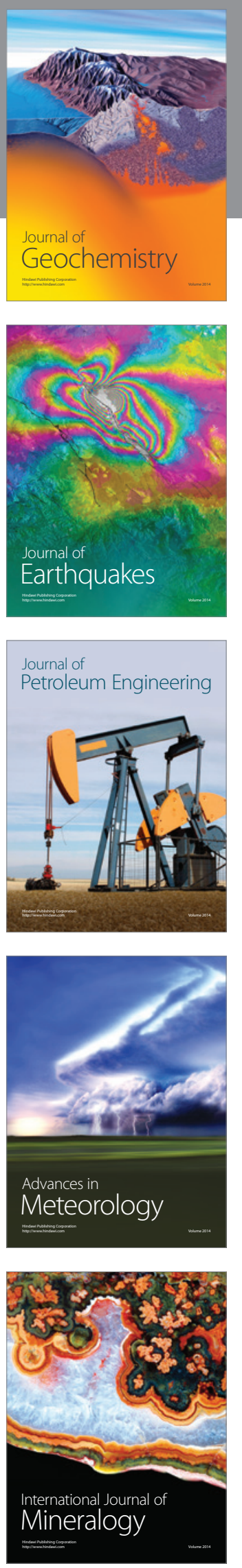
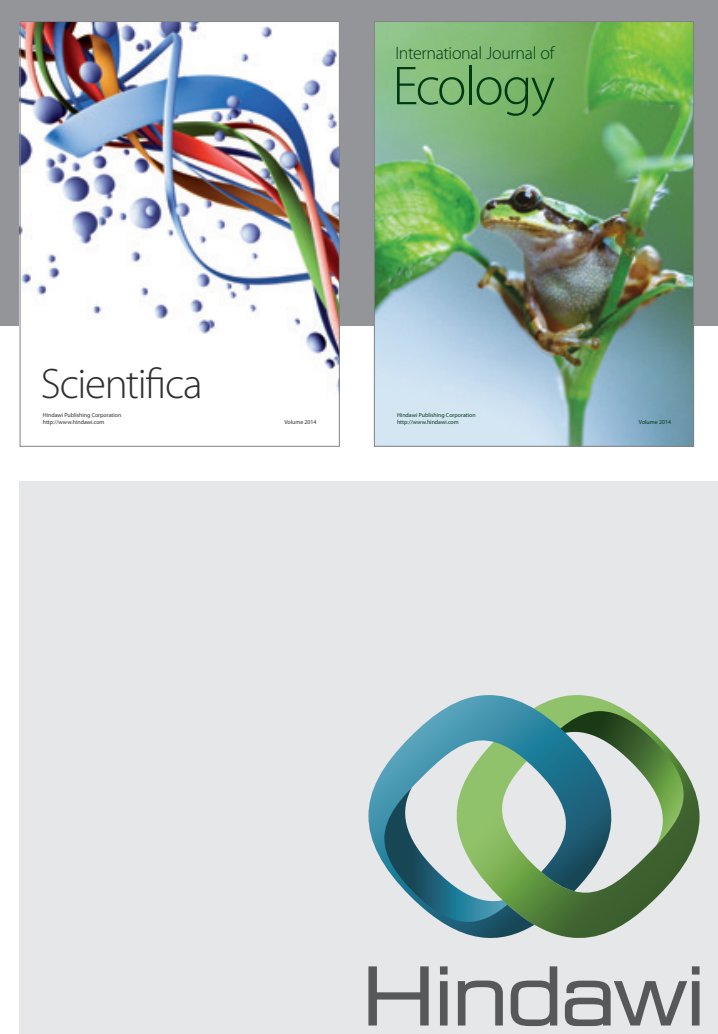

Submit your manuscripts at http://www.hindawi.com
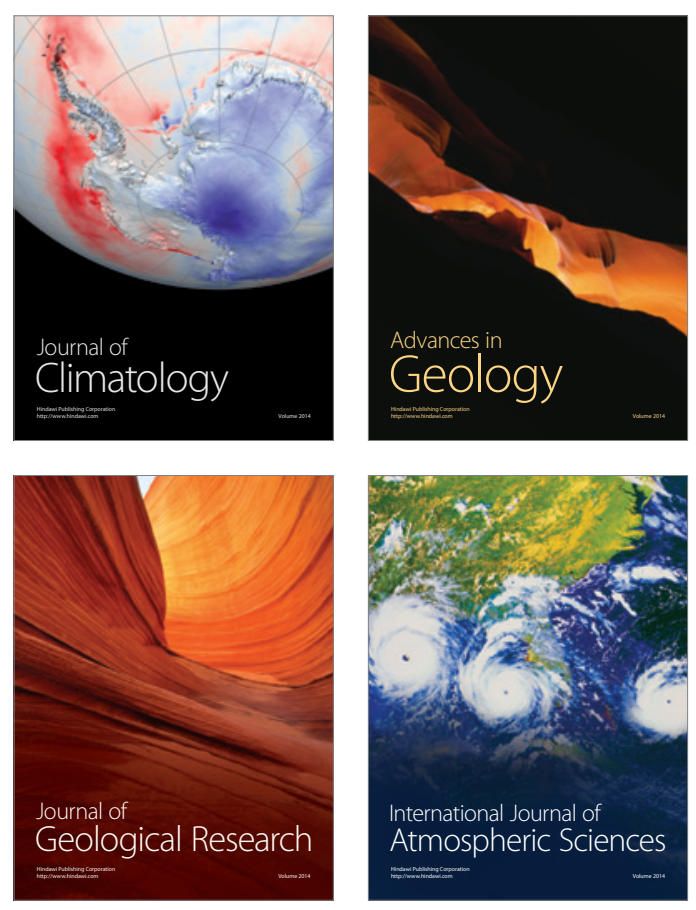
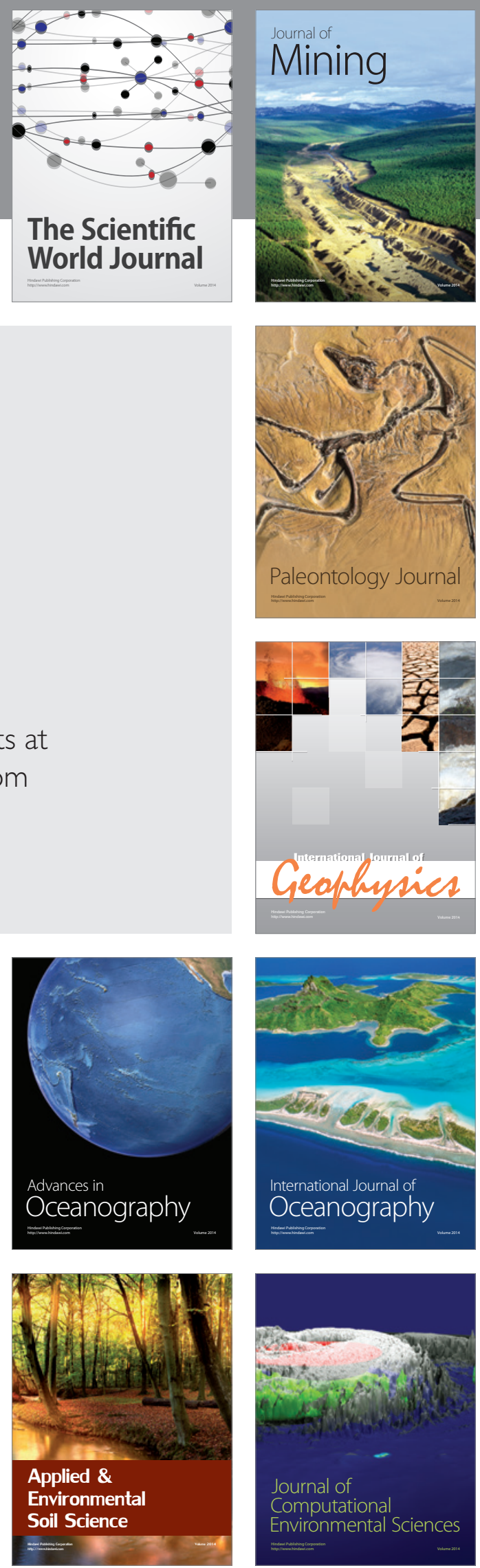